\title{
In Vitro Inhibition of Carboxylesterase 1 by Major Cannabinoids and Selected Metabolites
}

\author{
Yuli Qian, Xinwen Wang, and John S. Markowitz \\ Department of Pharmacotherapy and Translational Research, University of Florida, Gainesville, Florida (Y.Q., J.S.M.); and \\ Department of Clinical Pharmacy, University of Michigan, Ann Arbor, Michigan (X.W.)
}

Received December 22, 2018; accepted February 28, 2019

\section{ABSTRACT}

The escalating use of medical cannabis and significant recreational use of cannabis in recent years has led to a higher potential for metabolic interactions between cannabis or one or more of its components and concurrently used medications. Although there have been a significant number of in vitro and in vivo assessments of the effects of cannabis on cytochrome P450 and UDP-glucuronosyltransferase enzyme systems, there is limited information regarding the effects of cannabis on the major hepatic esterase, carboxylesterase 1 (CES1). In this study, we investigated the in vitro inhibitory effects of the individual major cannabinoids and metabolites $\triangle 9$-tetrahydrocannabinol (THC), cannabidiol (CBD), cannabinol (CBN), 11-nor-THC-carboxylic acid, and 11-hydroxy-THC on CES1 activity. $\$ 9$ fractions from human embryonic kidney 293 cells stably expressing CES1 were used in the assessment of cannabinoid inhibitory effects. THC, CBD, and CBN each exhibited substantial inhibitory potency, and were further studied to determine their mechanism of inhibition and kinetic parameters. The inhibition of CES1 by THC, CBD, and CBN was reversible and appears to proceed through a mixed competitivenoncompetitive mechanism. The inhibition constant $\left(K_{\mathrm{i}}\right)$ values for THC, CBD, and CBN inhibition were $0.541,0.974$, and $0.263 \mu \mathrm{M}$ $(0.170,0.306$, and $0.0817 \mu \mathrm{g} / \mathrm{ml})$, respectively. Inhibition potency was increased when THC, CBD, and CBN were combined. Compared with the potential unbound plasma concentrations attainable clinically, the $K_{\mathrm{i}}$ values suggest a potential for clinically significant inhibition of CES1 by THC and CBD. CBN, however, is expected to have a limited impact on CES1. Carefully designed clinical studies are warranted to establish the clinical significance of these in vitro findings.

\section{Introduction}

Cannabis [Cannabis sativa L. (marijuana)] is the most commonly abused substance globally and in the United States (http://apps.who.int/ iris/bitstream/handle/10665/251056/9789241510240-eng.pdf; jsessionid=28277FEC08D3255A96D4A8704EE2E1A3? sequence $=1$; https://www.samhsa.gov/data/report/2017-nsduh-detailed-tables). In 2017, an estimated 26 million Americans 12 years of age or older reported current (past month) marijuana use, a significant increase since 2002 (14.6 million) (https://www.samhsa.gov/data/report/2017-nsduh-detailed-tables). Beyond its widespread recreational use, the use of cannabis or one or more of its components for therapeutic purposes (i.e., "medical cannabis") has attracted much interest in recent years from both the lay public and the medical community. Medical cannabis is most commonly used to alleviate the symptoms (e.g., chronic pain, nausea, anorexia, seizure) associated with cancers, epilepsy, glaucoma, human immunodeficiency virus/AIDS, and others (Bridgeman and Abazia, 2017). As of November 2018 , the medical use of cannabis or its components in the United States was legal in 33 US states and the District of Columbia. Furthermore, in June 2018, the US Food and Drug Administration approved cannabidiol (CBD) oral solution for the treatment of seizures associated with two rare

https://doi.org/10.1124/dmd.118.086074. and severe forms of epilepsy, Lennox-Gastaut syndrome and Dravet syndrome, in patients 2 years of age and older (https://www.fda.gov/ newsevents/newsroom/pressannouncements/ucm611046.htm).

With increasing cannabis use, the potential for cannabis or one or more of its physically active constituents (cannabinoids) and their major metabolites interacting with other therapeutic agents also increases. This risk of drug-drug interactions (DDIs) could be substantial given that the diseases and conditions most frequently targeted for treatment with medical cannabis are chronic in nature and treated with conventional medications concurrently. A number of studies have been conducted to evaluate the effect of cannabis/cannabinoids on major drug-metabolizing enzymes. In vitro, the major cannabinoids $\Delta 9$-tetrahydrocannabinol (THC), CBD, and cannabinol (CBN) were found to either inhibit or induce the cytochrome P450 (P450) and UDP-glucuronosyltransferase (UGT) through various mechanisms and extents. Specifically, CYP3A4/5/7, CYP2D6, CYP2C9/19, CYP2A6, CYP2B6, CYP1A1/2, CYP1B1, and CYP2J2 were generally inhibited by those cannabinoids (Yamaori et al., 2010, 2011a,b,c, 2012; Jiang et al., 2013; Arnold et al., 2018), whereas CYP2C9 and the mRNA of CYP1A1 were induced by THC (Roth et al., 2001; Bland et al., 2005). As for UGT, UGT1A9 was inhibited by both CBD and CBN, and UGT2B7 was inhibited by CBD but was induced by CBN (Al Saabi et al., 2013). In clinical assessments, CBD oral solution was reported to significantly elevate blood/plasma/serum concentrations

ABBREVIATIONS: AUC, area under the plasma concentration-time curve; CBD, cannabidiol; CBN, cannabinol; CES1, carboxylesterase 1; DDI, drug-drug interaction; [I], inhibitor concentration; $I_{\max }$, maximal degree of inhibition; $I_{\text {max, } u}$, maximal unbound plasma concentration of the inhibitor observed in clinical studies; $K_{\mathrm{i}}$, inhibition constant; LC-MS/MS, liquid chromatography tandem mass spectrometry; OC, oseltamivir carboxylate; 11$\mathrm{OH}-\mathrm{THC}, 11$-hydroxy- $\Delta$ 9-tetrahydrocannabinol; OST, oseltamivir phosphate; P450, cytochrome P450; $R_{A \cup C}$, ratio of the area under plasma concentration-time curve of substrate with the presence of inhibitor over that without; $R_{v}$, percentage ratio; THC, $\triangle 9$-tetrahydrocannabinol; THC$\mathrm{COOH}, 11$-nor- $\Delta$ 9-tetrahydrocannabinol-carboxylic acid; UGT, UDP-glucuronosyltransferase. 
of antiepileptic drugs including clobazam, $N$-desmethylclobazam (the primary active metabolite of clobazam), rufinamide, topiramate, zonisamide, and eslicarbazepine (Geffrey et al., 2015; Gaston et al., 2017; Devinsky et al., 2018). In addition, chronic exposure to smoked cannabis was associated with decreases in the peak plasma concentration of indinavir and an increase in the clearance of theophylline (Jusko et al., 1978, 1979; Kosel et al., 2002), suggesting induction of the expression of CYP3A4 and CYP1A2.

Besides P450 and UGT enzymes, hydrolases are among the most essential enzymes catalyzing the metabolism of numerous frequently prescribed therapeutic agents (Williams et al., 2004; Laizure et al., 2013; Cerny, 2016). Human carboxylesterase 1 (CES1) is the predominant hepatic esterase, contributing to $80 \%-95 \%$ of total hydrolytic activity in human liver (Imai et al., 2006; Ross and Crow, 2007), and it hydrolyzes therapeutic agents from nearly every drug class formulated as carboxylic acid esters, amides, thioesters, and carbamates, among which specific agents may rely on CES1 for both deactivation/detoxification (e.g., methylphenidate) or metabolic activation (e.g., oseltamivir) (Takai et al., 1997; Satoh and Hosokawa, 2006; Laizure et al., 2013). CES1 also catalyzes the hydrolysis of a large number of endogenous compounds, including short- and long-chain acyl-glycerols, long-chain acyl-carnitine, and long-chain acyl-CoA esters (Lian et al., 2018). In vitro studies have reported significant inhibition of CES1 by specific therapeutic agents, suggesting a potential for clinically significant DDIs when used concurrently with other CES1 substrate medications (Fukami et al., 2010; Zhu et al., 2010; Rhoades et al., 2012; Thomsen et al., 2014). Additionally, an expanding number of natural products and traditional medicines have also been implicated as CES1 inhibitors (Wang et al., 2017).

Given the increasing use of medical and recreational cannabis, the potential for DDIs between cannabis and CES1 substrates warranted investigation. In the present study, we assessed the possible influence of three major cannabinoids (THC, CBD, and CBN) and two major metabolites [11-nor-THC-carboxylic acid (THC-COOH) and 11-hydroxy-THC (11-OH-THC)] (Fig. 1) on CES1 activity using an

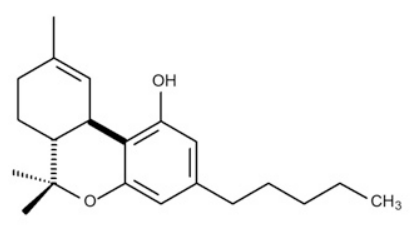

THC

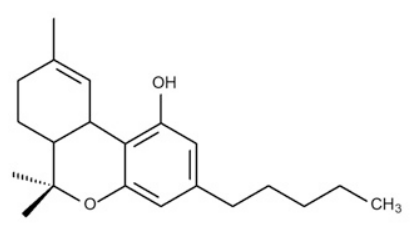

CBN

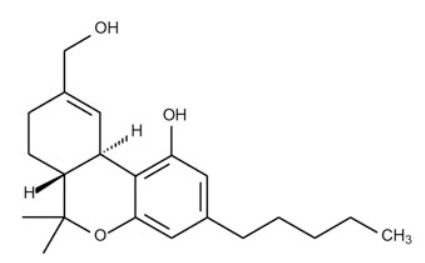

11-OH-THC

Fig. 1. Cannabinoids and selected metabolites chosen for assessment. in vitro CES1 assay system. Also investigated were the mechanisms and the in vitro potency of CES1 inhibition.

\section{Materials and Methods}

Materials. Oseltamivir phosphate (OST) was purchased from Sequoia Research Products Ltd. (Pangbourne, UK). Oseltamivir carboxylate (OC) was purchased from Toronto Research Chemicals Inc. (North York, ON, Canada). Ritalinic acid, montelukast, THC, CBD, CBN, THC-COOH, and 11-OH-THC were purchased from Cayman Chemical (Ann Arbor, MI). PBS and 1 M HEPES were purchased from Thermo Fisher Scientific Inc. (Waltham, MA). All other chemicals and reagents were of the highest analytical grade and were commercially available.

Preparation of Cell S9 Fractions Containing Wild-Type CES1. Human embryonic kidney cells (Flp-In-293; Invitrogen, Carlsbad, CA) stably expressing wild-type CES1 have been established and described previously (Zhu et al., 2008). Cells from the established cell line were cultured in Dulbecco's modified Eagle's medium with $10 \%$ FBS and $100 \mu \mathrm{g} / \mathrm{ml}$ hygromycin B until reaching approximately $90 \%$ confluence. Cells were then harvested in PBS and sonicated. Afterward, the cell lysates were centrifuged at $9000 \mathrm{~g}$ for 30 minutes at $4{ }^{\circ} \mathrm{C}$, from which the supernatant ( $\mathrm{S} 9$ fraction) was collected and stored at $-70^{\circ} \mathrm{C}$ in a freezer until use. The total protein concentrations in $\mathrm{S} 9$ fractions were determined using a Pierce BCA Protein Assay Kit (Thermo Fisher Scientific Inc.).

Metabolite Formation. The active OST metabolite OC, a product of OST hydrolysis (Fig. 2), was formed by incubating OST with S9 fractions in 2-ml tubes at a final volume of $100 \mu \mathrm{l}$. Various concentrations of substrate and S9 fractions were prepared separately in reaction buffers (PBS with $10 \mathrm{mM}$ HEPES). The reaction was initiated by the addition of substrate to $S 9$ fractions mixture in a water bath at $37^{\circ} \mathrm{C}$. Our preliminary study indicated that the formation of OC from OST was linear over S9 protein concentrations of $0-100 \mu \mathrm{g} / \mathrm{ml}$ and over incubation times of 0-30 minutes. Accordingly, a final S9 protein concentration of $20 \mu \mathrm{g} / \mathrm{ml}$ and a 15-minute incubation time were chosen as the standard reaction condition for ensuing inhibition studies. After incubation, the reaction was terminated by adding a 4-fold volume of methanol (400 $\mu \mathrm{l})$ containing $100 \mathrm{nM}$ ritalinic acid as the internal standard. The mixture was centrifuged at $16,100 \mathrm{~g}$ for 5 minutes, and the supernatant was further diluted with water/methanol before being transferred to high-performance liquid chromatography vials for liquid chromatography tandem mass spectrometry (LC-MS/MS) analysis.

Screening of Selected Cannabinoids and Their Metabolites as CES1 Inhibitors. A preliminary screening assay was conducted to identify the selected cannabinoids and metabolites for their potential to inhibit CES1 activity. THC, $\mathrm{CBD}, \mathrm{CBN}$, THC-COOH, and 11-OH-THC were screened at a single concentration of $5 \mu \mathrm{g} / \mathrm{ml}$. We have previously shown the leukotriene receptor antagonist montelukast to significantly inhibit CES1 activity, and it was used as a positive control $(10 \mu \mathrm{M})$ (Rhoades et al., 2012). The reaction was initiated by mixing S9 fractions (final concentration, $20 \mu \mathrm{g} / \mathrm{ml}$ ) with OST (final concentration, $500 \mu \mathrm{M}$ ) and tested compounds. The final incubation volume was $100 \mu \mathrm{l}$ with $1 \%$ DMSO. After incubation at $37^{\circ} \mathrm{C}$ for 15 minutes, the reactions were terminated and prepared for the LC-MS/MS analysis, as described in the Metabolite Formation section above.

Evaluation of Time-Dependent Inhibition by Selected Cannabinoids. To determine whether the inhibition of CES1 by any of the tested cannabinoids (THC, CBD, and CBN) is irreversible (mechanism-based), the inhibitory potency of those cannabinoids was evaluated with and without a 30-minute preincubation<smiles>CCOC(=O)C1=C[C@H](OC(CC)CC)[C@H](NC(C)=O)[C@H](N)C1</smiles>

Oseltamivir phosphate<smiles>CCC(CC)O[C@H]1C=C(C(=O)O)CC(N)[C@H]1NC(C)=O</smiles>

Oseltamivir carboxylate
Fig. 2. CES1-mediated activation of OST to OC. 
of them with S9 fractions before the addition of substrate (OST). The samples were divided into experimental and control groups. In the experimental samples, cannabinoid [final concentration, $0-50 \mu \mathrm{g} / \mathrm{ml}(0-161 \mu \mathrm{M})$, predissolved in $0.5-\mu \mathrm{l}$ DMSO because of its insolubility in aqueous buffer] was mixed with $\mathrm{S} 9$ fractions (final concentration, $20 \mu \mathrm{g} / \mathrm{ml}$ ) for a 30 -minute preincubation at $37^{\circ} \mathrm{C}$. After the preincubation, OST (final concentration, $1 \mathrm{mM}$ ) was added into the mixture to initiate the reaction. In the control group, samples were prepared in the same way as in the experimental group, except that cannabinoid was added together with OST after the preincubation. To avoid the potential influence introduced by a varied solvent (DMSO) effect among different samples, the final DMSO concentration $(\mathrm{v} / \mathrm{v})$ in all samples was adjusted to $0.5 \%$ during the preincubation and to $1 \%$ during the incubation. After incubation for 15 minutes, the reactions were terminated, and the analytical procedures were performed as described above in the Metabolite Formation section.

In Vitro Inhibition Study with Tested Cannabinoids. For determination of the inhibition constant $\left(K_{\mathrm{i}}\right)$ and the type of inhibition, kinetic studies were performed with varying concentrations of substrate $(0,100,500,1000,2500$, and $5000 \mu \mathrm{M}$ ) and tested cannabinoids (THC, CBD, and CBN concentration, $0-10 \mu \mathrm{g} / \mathrm{ml}[0-32.2 \mu \mathrm{M}]$ ). The premixture contained S9 fractions (final concentration, $20 \mu \mathrm{g} / \mathrm{ml}$ ) and cannabinoid in reaction buffer, and the reactions were initiated by adding substrate into the premixture. The final incubation volume was $100 \mu \mathrm{l}$ with $1 \%$ DMSO. After incubation at $37^{\circ} \mathrm{C}$ for 15 minutes, the samples were further prepared for the LC-MS/MS assay as described in the Metabolite Formation section above.

Assessment of CES1 Inhibition by Combined Cannabinoids. Since multiple cannabinoids are likely to present in the systemic circulation and liver after the more common types of cannabis use and routes of administration (e.g., smoking), we further assessed whether the inhibition on CES1 would be increased by the combination of the major cannabinoid constituents THC, CBD, and CBN.
The final concentrations of $\mathrm{THC}, \mathrm{CBD}$, and $\mathrm{CBN}$ used in experiments were $0.200(0.636 \mu \mathrm{M}), 0.133(0.424 \mu \mathrm{M})$, and $0.133 \mu \mathrm{g} / \mathrm{ml}(0.430 \mu \mathrm{M})$, respectively. Those concentrations were approximated with consideration of their calculated $K_{\mathrm{i}}$ values and potentially achievable plasma concentrations reported in different clinical studies (Ohlsson et al., 1986; Johansson et al., 1987; Huestis et al., 1992). Cannabinoids were prepared in glass vials before mixing with $\mathrm{S} 9$ fractions (final concentration, $20 \mu \mathrm{g} / \mathrm{ml}$ ) to minimize absorption into tube walls. The reaction was initiated by adding OST $(500 \mu \mathrm{M})$ to a premixture of S9 fractions and cannabinoids. The final incubation volume was $100 \mu 1$ with $1 \%$ DMSO. After a 15 -minute incubation at $37^{\circ} \mathrm{C}$, the samples were prepared for LC-MS/MS assay as described in the Metabolite Formation section above. The degree of inhibition was compared between samples containing individual cannabinoids versus the three combined cannabinoids.

LC-MS/MS Analysis. OC, the active hydrolytic metabolite of OST, was determined using a high-performance liquid chromatography system (Shimadzu, Kyoto, Japan) coupled to an AB Sciex API 3000 Triple-Quadrupole Mass Spectrometer (Applied Biosystems, Foster City, CA). Chromatographic separation was achieved on a C18 reverse-phase analytic column (Aqua, $50 \times 2.0 \mathrm{~mm}, 5 \mu \mathrm{m}$; Phenomenex Inc., Torrance, CA). A gradient mobile phase was used, with the aqueous phase containing $0.1 \%$ formic acid in water, and the organic phase containing methanol. The mobile phase was delivered at a total flow rate of $0.3 \mathrm{ml} / \mathrm{min}$. Mass spectrometric analysis was performed via electrospray ionization in positive mode, and the mass transitions of mass/charge ratios $285.3 \rightarrow 138.3$ and $220.2 \rightarrow 84.4$ were monitored for OC and ritalinic acid, respectively. The lower limit of quantification of $\mathrm{OC}$ was estimated to be $25 \mathrm{nM}$. The interday and intraday assay precision as well as accuracy were within $10 \%$. A representative chromatogram obtained from the incubation of S9 fractions with $100 \mu \mathrm{M}$ OST was shown in Fig. 3.

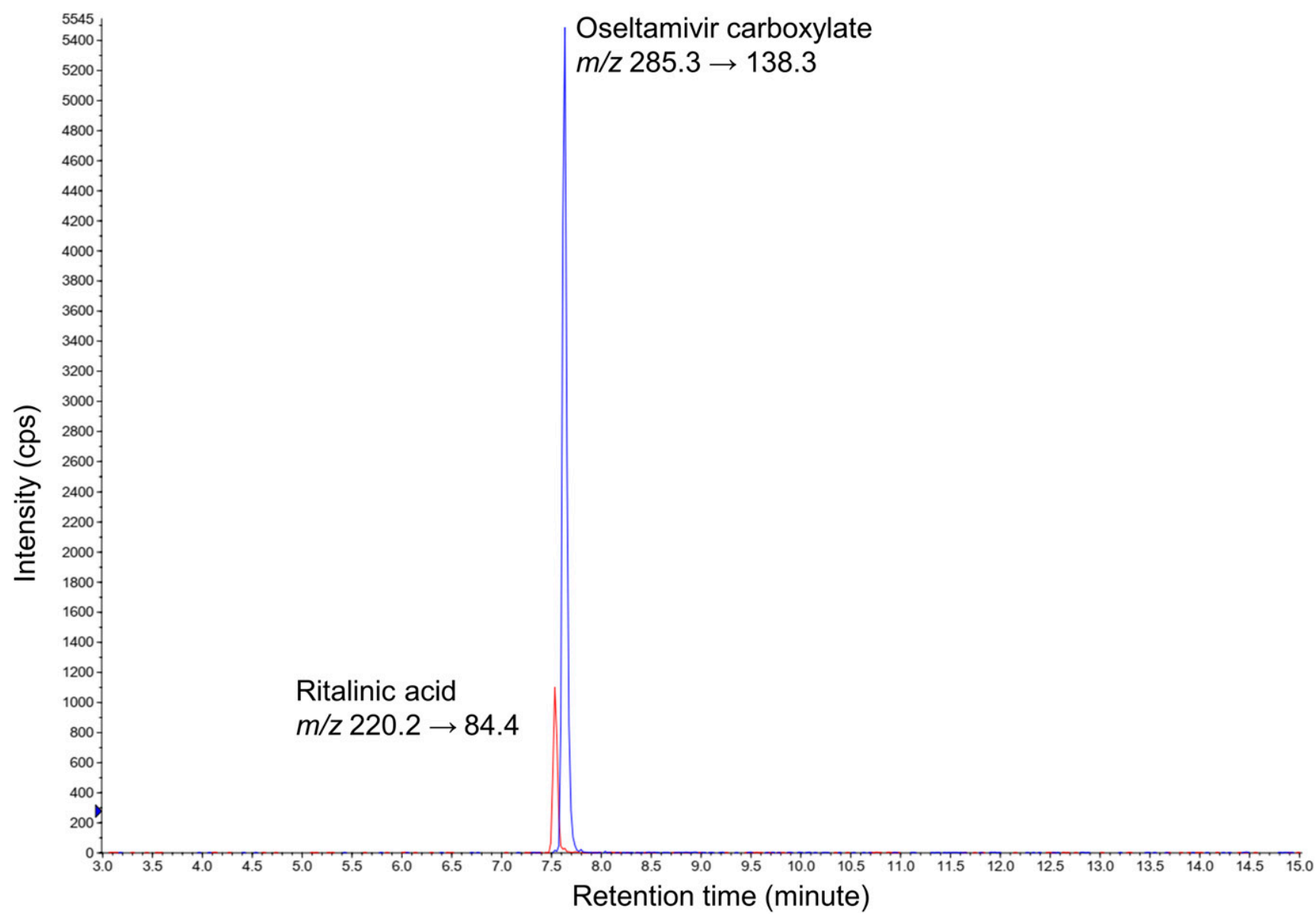

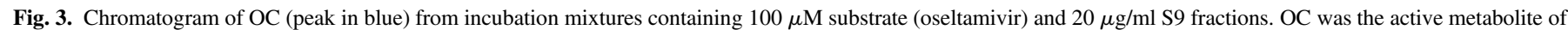
oseltamivir specifically catalyzed by CES1. Ritalinic acid (peak in red) was used as the internal standard. $\mathrm{m} / \mathrm{z}$, mass/charge ratio. 
Data Analysis. In accordance with a previous report (Hoffmann et al., 2009), some spontaneous hydrolysis of OST in aqueous buffer was observed in our study. Therefore, in the kinetic analysis, the metabolite amount formed in control samples without $\mathrm{S} 9$ fractions was subtracted from that in samples with the same substrate concentrations.

In the time-dependent inhibition study, the metabolite (OC) formation rate was expressed as a percentage ratio $\left(R_{v}\right)$ of the rate in the control sample without inhibitor. The independent variable was inhibitor concentration ([I]). The halfmaximal inhibition concentration (IC) was estimated by fitting the following equation (eq. 1) into the experimental data using nonlinear regression:

$$
R_{v}=100 \times\left(1-\frac{I_{\max } \cdot[I]^{b}}{[I]^{b}+I C^{b}}\right) .
$$

Additional iterated parameters were as follow: $I_{\max }$, the maximal degree of inhibition; and $b$, a shape exponent. The $\mathrm{IC}_{50}$ (i.e., $[I]$ that achieves $50 \% R_{v}$ ) was calculated as follows (eq. 2):

$$
I C_{50}=I C /\left(2 I_{\max }-1\right)^{1 / b} .
$$

In the in vitro inhibition study, the metabolite formation rate $(V)$ was expressed as the dependent variable of the substrate concentration $([S])$ and $[I]$. A mixed competitive-noncompetitive inhibition model derived from Michaelis-Menten kinetics (eq. 3) was used to evaluate the type of inhibition and to determine the $K_{\mathrm{i}}$ values of tested cannabinoids. Nonlinear regression analysis was performed to fit the model into the experimental data:

$$
V=\frac{V_{\max } \cdot[S]}{K_{m}\left(1+\frac{[I]}{K_{i}}\right)+[S]\left(1+\frac{[I]}{\alpha \cdot K_{i}}\right)}
$$

Iterated parameters were as follows: $K_{\mathrm{m}}$, the Michaelis-Menten constant; $K_{\mathrm{i}}$ for inhibitor; and $\alpha$, an indicator of the inhibition type (an $\alpha$ approaching positive infinity indicates competitive inhibition, an $\alpha$ equal to 1 indicates noncompetitive inhibition, and an $\alpha$ falling in between indicates mixed inhibition). The majority of notations of kinetic parameters and symbols are adapted from the recommendations of Segel (1975).

The type of inhibition was also verified by visual inspection of LineweaverBurk plots of the experimental data. The data points at the lowest OST concentration $(100 \mu \mathrm{M})$ were not shown in the plots for easier visual assessment of the line cross point and thereby the inhibition type. In addition, the data group with the highest $\mathrm{CBN}$ concentration $(10 \mu \mathrm{g} / \mathrm{ml}[32.2 \mu \mathrm{M}])$ was excluded because too few metabolites were formed.

The potential of clinical DDIs was assessed using the predicted ratio of the area under plasma concentration-time curve (AUC) of substrate with the presence of inhibitor over that without $\left(R_{A U C}\right)$. The predicted ratio (RAUC) was calculated as follows (eq. 4):

$$
R_{A U C}=1+I_{\max , u} / K_{i}
$$

where $I_{\max , u}$ is the maximal unbound plasma concentration of the inhibitor observed in clinical studies, and $K_{\mathrm{i}}$ is the estimated $K_{\mathrm{i}}$ in vitro.

Software and Statistical Methods. The parameter estimation was performed in R 3.4.2 (R Foundation for Statistical Computing, Vienna, Austria) using the "nls" package with a least squares approach. The estimation algorithm was GaussNewton. The model performance was assessed by diagnostic residual plots, visual check, and the certainty of parameter estimates. Data visualization was performed in R 3.4.2 using the "ggplot2" package. Lineweaver-Burk plots were made using Excel (Microsoft, Redmond, WA).

\section{Results}

Screening of Selected Cannabinoids and Their Metabolites. The relative formation velocities of OC in experimental samples were expressed as a percentage of the results observed in the negative control containing only S9 fractions and substrate. Decreased velocities were observed for all tested compounds (THC, CBD, CBN, THC-COOH, and 11-OH-THC), indicating their inhibitory effects on CES1 (Fig. 4). At a concentration of $5 \mu \mathrm{g} / \mathrm{ml}$, inhibition of greater than $90 \%$ was observed for THC, CBD, and CBN, whereas only less than 50\% CES1 inhibition was observed for THC-COOH and 11-OH-THC. Considering the degree of systemic exposures to all of the tested compounds in humans after cannabis/cannabinoid administration, only THC, CBD, and CBN were further investigated.

THC, CBD, and CBN Reversibly Inhibited Oseltamivir Hydrolysis. The formation rates of OC from OST were decreased by THC, CBD, and CBN in a concentration-dependent manner (Fig. 5). The $\mathrm{IC}_{50}$ for each cannabinoid was computed and compared between samples in which the $\mathrm{S} 9$ fractions were preincubated with cannabinoids for 30 minutes before the addition of substrate (Fig. 5A), and those in which cannabinoids were added after the preincubation (Fig. 5B). A 30-minute preincubation with any of the tested cannabinoids did not increase their inhibition potency (Table 1), indicating that their inhibition on CES1-mediated OST hydrolysis was not time dependent.

In Vitro Kinetic Study on Inhibition of CES1 Activity by Selected Cannabinoids. An in vitro study was conducted at varying concentrations of substrate (OST, 0-5000 $\mu \mathrm{M}$ ) and tested cannabinoids $[0-10 \mu \mathrm{g} / \mathrm{ml}(0-32 \mu \mathrm{M})]$. Nonlinear regression analysis was performed to characterize the type of inhibition and estimate kinetic parameters (Fig. 6, A1, B1, and C1), which were summarized in Table 2. The $K_{\mathrm{i}}$ values for THC, CBD, and $\mathrm{CBN}$ inhibition of OST hydrolysis were $0.541,0.974$, and $0.263 \mu \mathrm{M}(0.170,0.306$, and $0.0817 \mu \mathrm{g} / \mathrm{ml})$, respectively. Based on the $K_{\mathrm{i}}$ values, the rank order of in vitro inhibition potency of the tested cannabinoids was $\mathrm{CBN}>\mathrm{THC}>\mathrm{CBD}$. The values of $\alpha$ and Lineweaver-Burk plots (Fig. 6, A2, B2, and C2) indicated that the inhibition of CES1 by all three cannabinoids followed a mixed competitive-noncompetitive model, with characteristics more closely resembling a noncompetitive model.

Increased Inhibition by Combined THC, CBD, and CBN. When THC, CBD, and CBN were incubated individually and in combination with CES1, the combined cannabinoids exhibited a higher extent of inhibition than that observed in any individual group (Fig. 7).

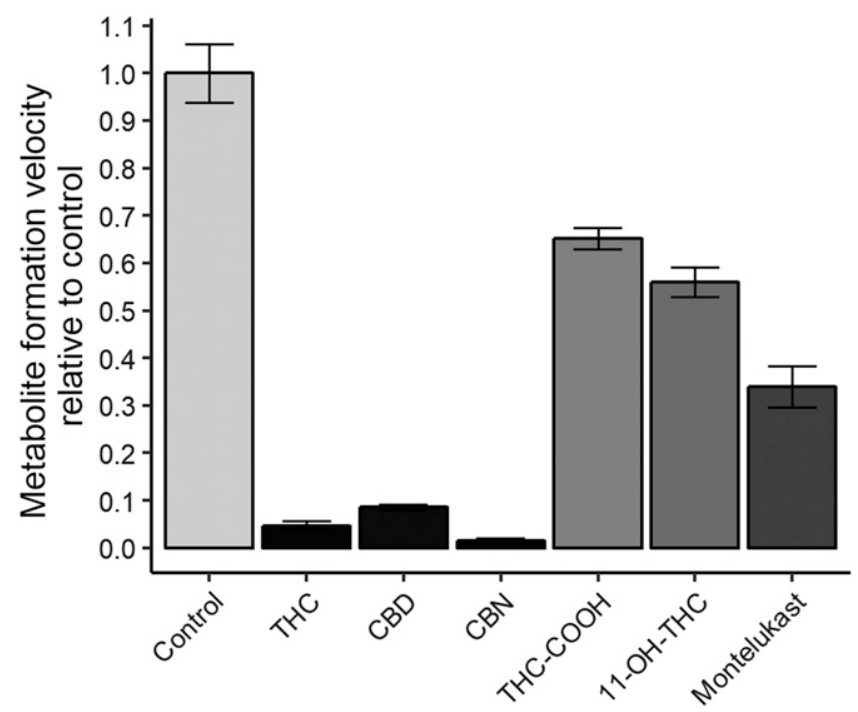

Fig. 4. Screening of selected cannabinoids and their metabolites as potential modifiers of CES1 activity. The substrate (oseltamivir) concentration was fixed at $500 \mu \mathrm{M}$. The concentration of all tested compounds was $5 \mu \mathrm{g} / \mathrm{ml}$. Montelukast $(10 \mu \mathrm{M})$ served as a positive control. The CES1 activity was expressed as a ratio relative to the control containing no tested compounds. Individual bars represent the mean ( \pm S.D.) of triplicate samples. The shading of the bars indicates the extent of CES1 inhibition. 

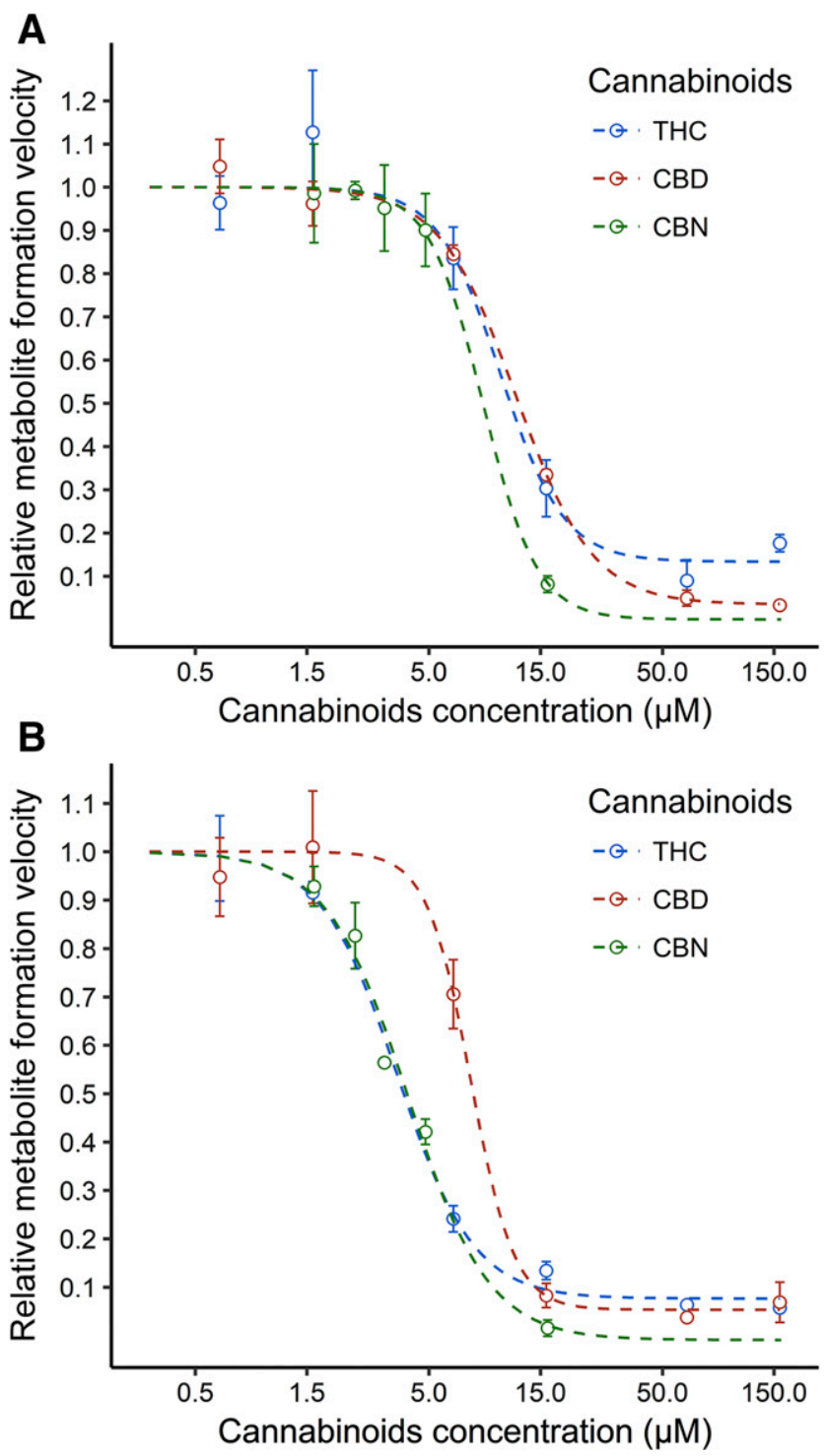

Fig. 5. Inhibition curves for tested cannabinoids in a time-dependent inhibition study. The tested cannabinoids were added into S9 fractions before (A) and after (B) a 30-minute preincubation. The substrate (oseltamivir) concentration was fixed at $1000 \mu \mathrm{M}$. The reaction velocity was expressed as a ratio relative to the control containing no inhibitor. Individual points represent the mean $( \pm$ S.D.) of triplicate samples.

\section{Discussion}

Increased medical and recreational use of cannabis/cannabinoids and concurrent use of conventional medications poses a risk of DDI. We evaluated the potential for major cannabinoids and selected metabolites to inhibit CES1. We identified the three major cannabinoids, THC, CBD, and CBN, as potent inhibitors of CES1 in vitro. Preincubation of $\mathrm{S} 9$ fractions with each of the compounds did not result in the potentiation of inhibition, indicating reversible characteristics. Furthermore, the inhibition mechanisms were revealed via the parameter $\alpha$ using the reversible inhibition model (eq. 3) as well as visual inspection of Lineweaver-Burk plots. The estimated $\alpha$ values for the inhibition of CES1 by THC, CBD, and $\mathrm{CBN}$ were 14.0, 5.8, and 12.0, respectively (Table 1), demonstrating that the inhibition type was mixed competitive-noncompetitive with characteristics more closely resembling noncompetitive inhibition for all three cannabinoids. Lineweaver-Burk plots confirmed this result.

The potential for clinically relevant DDI between tested cannabinoids and CES1 substrates was evaluated comparing the in vitro $K_{\mathrm{i}}$ from
TABLE 1

Effect of preincubation with tested cannabinoids on CES1 activity

\begin{tabular}{|c|c|c|c|}
\hline \multirow{3}{*}{ Cannabinoids } & \multicolumn{2}{|c|}{$\mathrm{IC}_{50}$} & \multirow{3}{*}{$\mathrm{B} / \mathrm{A}$} \\
\hline & \multicolumn{2}{|c|}{ Preincubation time } & \\
\hline & $0 \min (\mathrm{A})$ & $30 \min (B)$ & \\
\hline & \multicolumn{2}{|c|}{$\mu \mathrm{M}$} & \\
\hline $\mathrm{THC}$ & 3.91 & 11.2 & 2.85 \\
\hline CBD & 7.73 & 12.1 & 1.57 \\
\hline CBN & 4.03 & 8.51 & 2.11 \\
\hline
\end{tabular}

All $\mathrm{IC}_{50}$ estimates were obtained from nonlinear regression analysis using the model described in eq. 1 .

our study results to the potentially achievable $[I]$ values in humans. A predicted change $\left(R_{A U C}\right)$ in the substrate drug AUC by tested cannabinoids was calculated by eq. 4 . According to both the Food and Drug Administration and the European Medicines Agency, an $R_{A U C}$ value larger than 1.02 warrants further investigations into the DDI potential using either mechanistic models or formal clinical study (https://www.ema.europa.eu/documents/scientific-guideline/ guideline-investigation-drug-interactions_en.pdf; https://www.fda.gov/ ucm/groups/fdagov-public/@fdagov-drugs-gen/documents/document/ ucm581965.pdf). The exposure to the various cannabis constituents as determined by blood sampling of the systemic circulation varies greatly and is influenced by the formulation used, dosing routes (e.g., oral vs. sublingual vs. smoking vs. vaporizing), and dosing schedule (Huestis, 2007). Maximal exposure scenarios were assumed in our evaluation where the highest observed plasma concentrations of tested cannabinoids were used. Smoking, the most common route of cannabis use, generally renders the highest THC exposures relative to vaporized and orally ingested cannabis/cannabinoids (Grotenhermen, 2003; Huestis, 2007; Lucas et al., 2018). In a clinical study, the $C_{\max }$ values of THC in six healthy males averaged $162 \mathrm{ng} / \mathrm{ml}(0.515 \mu \mathrm{M})$ after smoking a cannabis cigarette containing 3.55\% THC (Huestis et al., 1992). Similar THC concentration ranges were observed in other studies where THC was administered by smoking cannabis cigarettes (Lindgren et al., 1981; Perez-Reyes et al., 1982; Lee et al., 2015). The contents of CBD and CBN in cannabis preparations were much lower than that of THC (average: 4.5\% THC, $0.4 \% \mathrm{CBD}$, and $0.3 \% \mathrm{CBN}$ in cannabis during 1993-2008) (Mehmedic et al., 2010). After smoking a cigarette containing $19 \mathrm{mg}$ of deuterium-labeled CBD, a mean $C_{\max }$ of $110 \mathrm{ng} / \mathrm{ml}(0.350 \mu \mathrm{M})$ was observed in five healthy males (Ohlsson et al., 1986). In a more recently published pharmacokinetic study on CBD oral solution (Epidiolex) (Taylor et al., 2018), a single dose of 1500-6000 mg was found to be well tolerated in six healthy subjects in each dose arm, and the mean $C_{\max }$ of CBD was measured to be $292-782 \mathrm{ng} / \mathrm{ml}(0.928-2.49 \mu \mathrm{M})$. There is a dearth of information available on $\mathrm{CBN}$ pharmacokinetics in humans, with a few studies reporting low $[<12 \mathrm{ng} / \mathrm{ml}(0.0387 \mu \mathrm{M})]$ or unquantifiable (less than the lower limit of quantification) blood/plasma concentrations after smoked or vaporized doses (Schwope et al., 2011; Desrosiers et al., 2014; Newmeyer et al., 2016). Johansson et al. (1987) reported a mean $\mathrm{CBN} C_{\max }$ of $126 \mathrm{ng} / \mathrm{ml}(0.406 \mu \mathrm{M})$ in six healthy males after smoking a cannabis cigarette containing $19 \mathrm{mg}$ of CBN. However, since CBN is unlikely to be administered or used therapeutically in a relatively pure form, this concentration was not considered to be clinically achievable for purposes of our assessment. In the $R_{A U C}$ calculation, the plasma protein binding of THC and CBD was assumed to be $97.2 \%$ and $94 \%$, respectively, based on available data (Garrett and Hunt, 1974; https://www.accessdata.fda.gov/drugsatfda_docs/ nda/2018/210365Orig1s000ClinPharmR.pdf). We were unable to locate documentation of CBN plasma protein binding, so an empirical range 


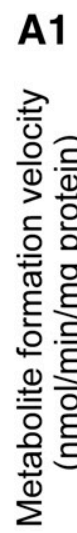

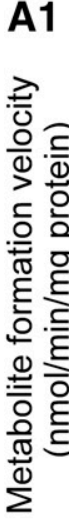

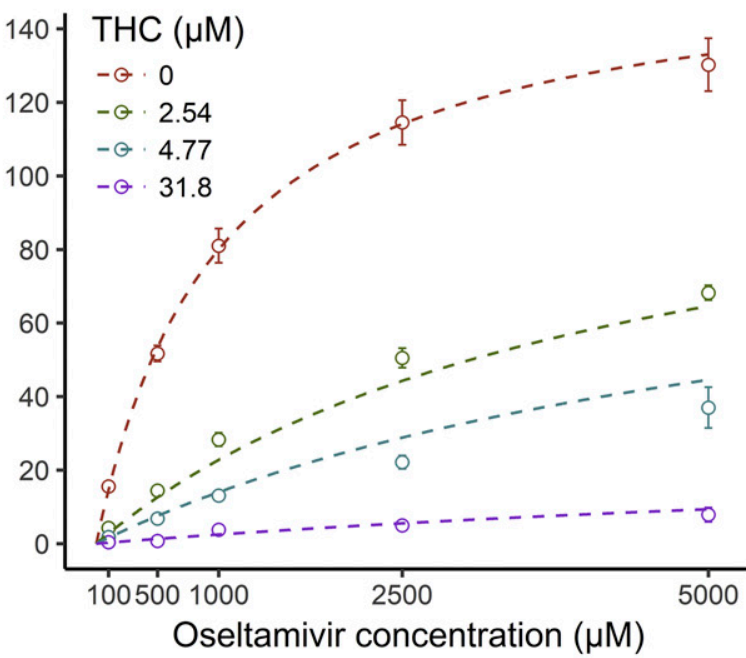

B1

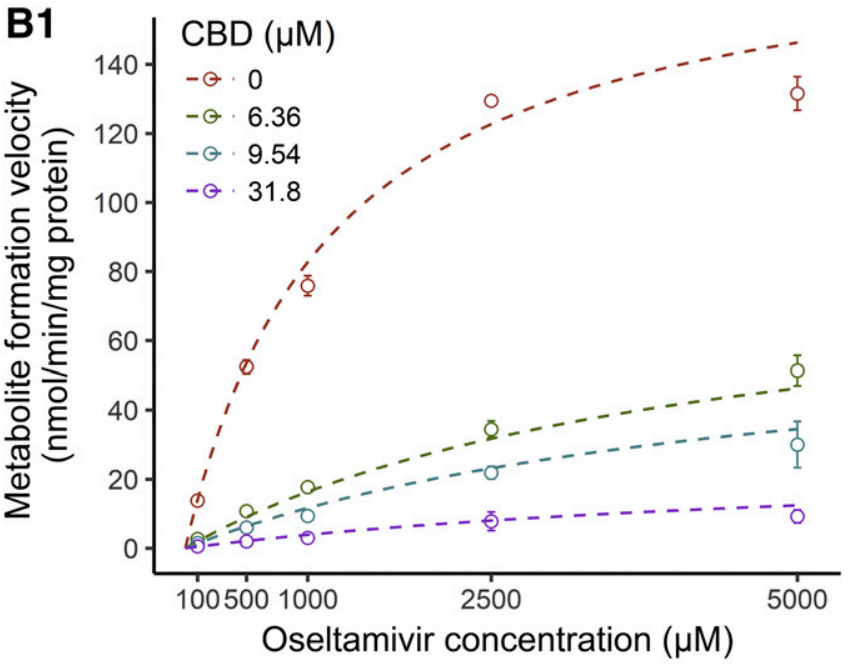

C1

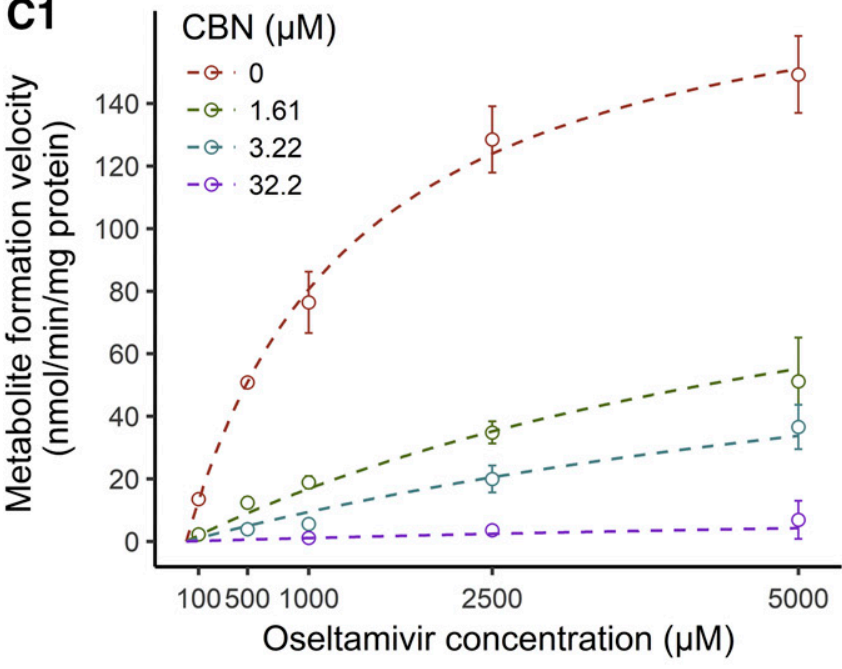

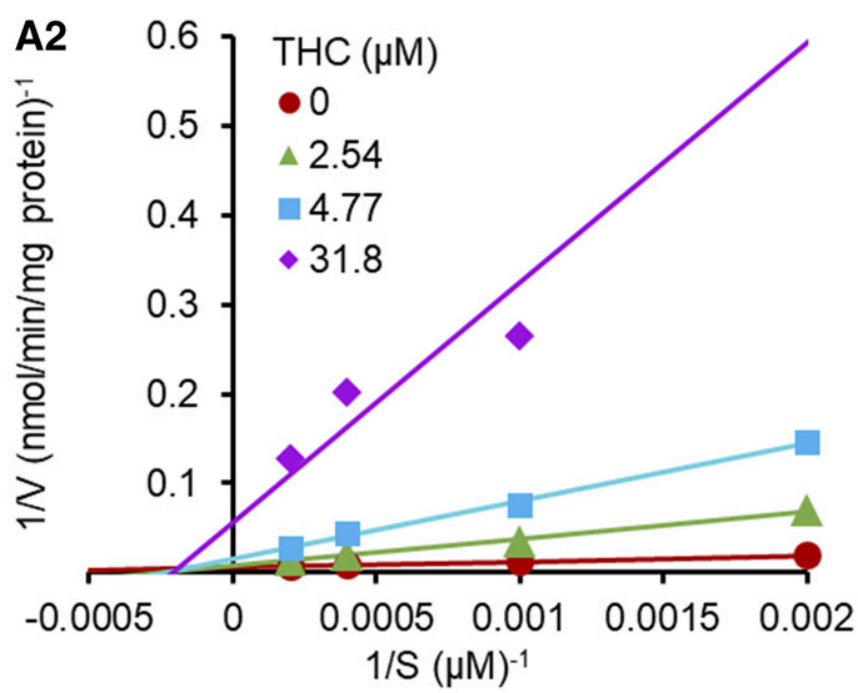
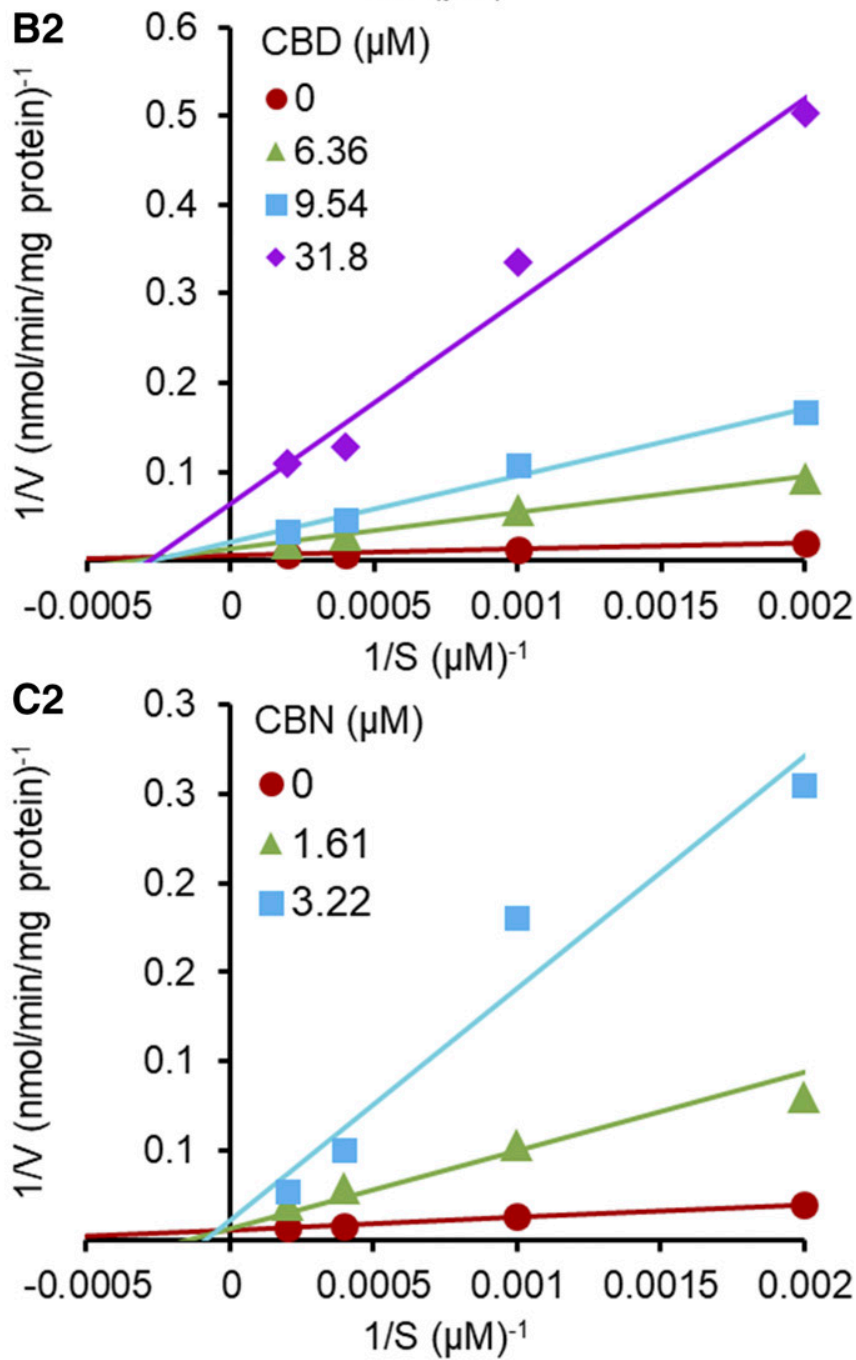

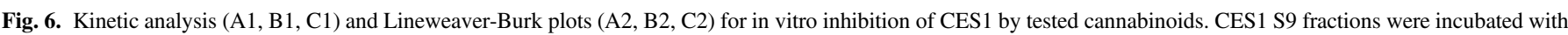

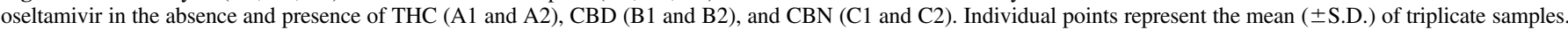

of $>90 \%$ was chosen given its similarity in structure and lipophilicity to THC and CBD. Taken altogether, the $I_{\max , u}$ (eq. 4) was calculated as: $\left(C_{\max }\right) \times(1-$ plasma protein binding $)$, and the resulting RAUC for THC, CBD, and CBN were 1.027, 1.057-1.153, and $<1.015$, respectively. These results suggest that THC and CBD may act as "perpetrator" constituents toward CES1 substrates, but this requires further clinical investigation and confirmation. Regarding CBN, which also exhibited potent in vitro inhibitory effects on CES1, it appears less likely to participate in clinically significant DDIs due to its low anticipated exposure in individuals using cannabis for recreational or medical purposes. 
TABLE 2

Parameter estimates of in vitro inhibition study with tested cannabinoids

\begin{tabular}{lrrrc}
\hline Cannabinoids & \multicolumn{1}{c}{$K_{\mathrm{m}}$} & \multicolumn{1}{c}{$K_{\mathrm{i}}$} & $\alpha$ & $\mathrm{V}_{\max }$ \\
\hline \multicolumn{3}{c}{$\mu \mathrm{M}$} \\
THC & $993 \pm 149$ & $0.541 \pm 0.081$ & $14.0 \pm 9.3$ & nmol/min per milligram of protein \\
CBD & $1191 \pm 159$ & $0.974 \pm 0.169$ & $5.8 \pm 3.4$ & $159 \pm 10$ \\
CBN & $1399 \pm 108$ & $0.263 \pm 0.034$ & $12.0 \pm 9.8$ & $181 \pm 9$ \\
& & &
\end{tabular}

$K_{\mathrm{m}}$, Michaelis-Menten constant; $\alpha$, an indicator of inhibition type; $\mathrm{V}_{\max }$, the maximum metabolite formation velocity.

All values were presented as the parameter estimates \pm S.E. (uncertainty) from nonlinear regression analysis using the model described in eq. 3 .

Our study also demonstrated increased inhibition of CES1 activity when THC, CBD, and CBN were combined versus individual constituents. Since individuals using cannabis or multiconstituent extracts for medical reasons or on a recreational basis are more likely to be exposed to a number of major cannabinoids rather than single constituents, this approach may provide a better assessment of potential DDI liability.

Exposures to cannabis can vary depending on product content, dosing route, and schedule. Even within a single dosing route such as smoking, multiple factors influence cannabinoid exposure, including the cannabis potency; number, duration, and spacing of puffs; hold time; and inhalation volume (Huestis, 2007). Further, substantial intrasubject and intersubject variability in cannabinoid exposures has been documented in pharmacokinetic studies. In the present study, we estimated the clinical DDI potential by adopting the higher values we found reported in published literature, which might be achieved in actual use scenarios.

A further assumption made in our study was that hydrolysis rates of OST represented only CES1 catalytic activity. This was a reasonable assumption since there is minimal native expression of drug-metabolizing enzymes, including other esterases in the parent embryonic kidney 293 cells from which the CES1-overexpressing cells were made (Bouman et al., 2011). In addition, OST was reported not to interact with either P450s or UGTs (He et al., 1999), which further validated our assumption.

Our study has several limitations. First, like most in vitro assessments, our evaluation and interpretation were based only on reported systemic concentrations of cannabinoids rather than on actual tissue

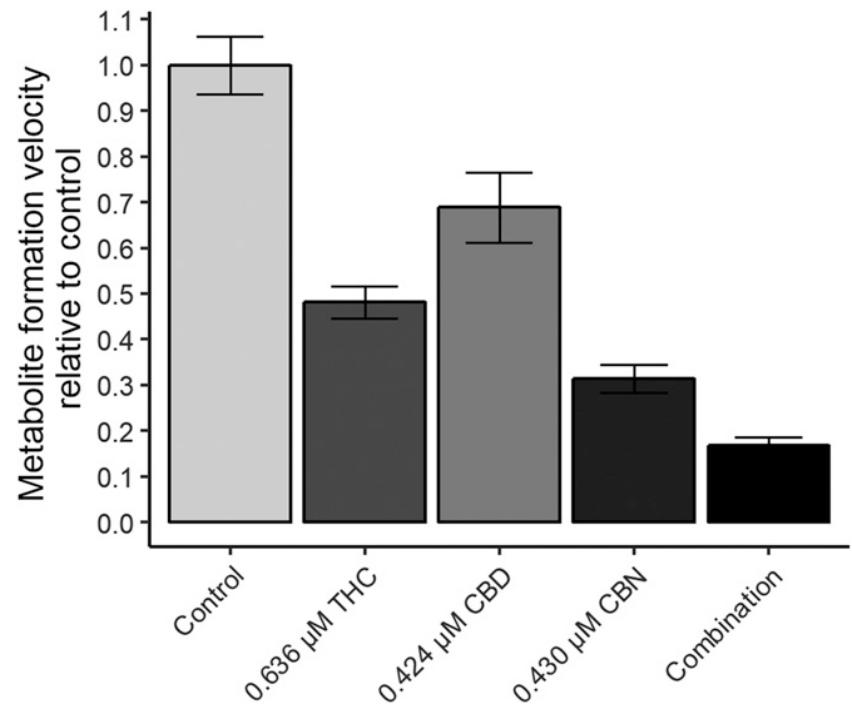

Fig. 7. Inhibition effects of combined cannabinoids on CES1 activity. The substrate (oseltamivir) concentration was fixed at $500 \mu \mathrm{M}$. The final concentrations of cannabinoids in the combination sample were $0.363 \mu \mathrm{M}$ THC, $0.424 \mu \mathrm{M} \mathrm{CBD}$, and $0.430 \mu \mathrm{M}$ CBN. The CES1 activity was expressed as a ratio relative to the control containing no inhibitors. Individual bars represent the mean $( \pm$ S.D.) of triplicate samples. The shading of the bars indicates the extent of CES1 inhibition. concentrations. CES1 is a ubiquitously expressed enzyme with the liver being by far the predominant expression site (Satoh et al., 2002; Fagerberg et al., 2014). Assessing DDIs based on concentrations reaching metabolic sites (i.e., liver) could generally yield a more accurate prediction. However, there are almost no data regarding cannabinoid hepatic concentrations in humans, beyond limited postmortem data, suggesting that certain cannabinoids (i.e., $\mathrm{CBD}, \mathrm{CBN}$ ) attain much higher concentrations in the liver and bile relative to systemic concentrations (Gronewold and Skopp, 2011; Fabritius et al., 2012). Second, in the inhibition study conducted for $K_{\mathrm{i}}$ determination, all $[I]$ values used were higher than the estimated $K_{\mathrm{i}}$ values. We believe that the relatively large uncertainty around the estimated $\alpha$ values was partially due to this limitation, and it could potentially introduce some error in the prediction of inhibition at lower $[I]$ values. However, the concentrations of THC, CBD, and CBN in the combination assessment were in the range of their $K_{\mathrm{i}}$ values, and the inhibition results were consistent with our model prediction, which increased the confidence in estimated parameters.

Reversible inhibition was concluded for THC, CBD, and CBN since their $\mathrm{IC}_{50}$ did not decrease after a 30-minute preincubation with $\mathrm{S} 9$ fractions. On the contrary, an actual increase of $\mathrm{IC}_{50}$ after the preincubation was observed. This phenomenon has also been described in previous reports by another group (Yamaori et al., 2010, 2011c, 2012). The exact causes of this phenomenon are unclear. As a technical note, we have observed that when prepared at lower concentrations (i.e., around their $K_{\mathrm{i}}$ values), THC, CBD, and CBN prepared in plastic tubes exhibited lower inhibition potency compared with finding from experiments using glass vials. Therefore, absorption of the cannabinoids onto the plastic incubation tube walls was believed to be a likely cause. This phenomenon has previously been noted by other investigators (Garrett and Hunt, 1974; Christophersen, 1986). Therefore, the incubation time was restricted to 15 minutes to minimize this potential influence.

Finally, an additional issue for consideration of our findings relates to the role of CES1 in the biotransformation of a large number of endogenous compounds. Indeed, CES1-mediated hydrolysis of esters (e.g., cholesteryl esters and triacylglycerols) is increasingly viewed as having an important role in lipid metabolism, cholesterol homeostasis, and possibly fatty liver disease (Lian et al., 2018). Though speculative, a sustained inhibition of CES1 activity as a result of chronic exposure to cannabinoids could have implications for these biologic processes as well.

In summary, our study identified THC, $\mathrm{CBD}$, and $\mathrm{CBN}$ as potent inhibitors of CES1 in vitro, with a reversible inhibition mechanism and mixed competitive-noncompetitive characteristics. The calculated $K_{\mathrm{i}}$ values for THC, CBD, and CBN were 0.541, 0.974, and $0.263 \mu \mathrm{M}$, respectively. An increase in the inhibition potency was observed when THC, CBD, and CBN were combined for assessment. When compared with the respective cannabinoid $C_{\max }$ values achievable physiologically, the $K_{\mathrm{i}}$ values suggest that DDI may occur after the concomitant administration of CES1 substrate medications and THC and CBD, but not after administration of CBN. 
The determination of whether the observed in vitro inhibitory effects of THC and CBD translates into significant, or even detectable, clinical DDIs requires further investigation through formal clinical study. Such formal clinical studies would require the utilization of a well-characterized cannabis product, a standardized dosing regimen, and incorporation of an appropriate CES1 substrate as a probe therapeutic agent.

\section{Authorship Contributions}

Participated in research design: Qian, Wang, Markowitz.

Conducted experiments: Qian.

Performed data analysis: Qian, Wang, Markowitz.

Wrote and contributed to the writing of the manuscript: Qian, Markowitz.

\section{References}

Al Saabi A, Allorge D, Sauvage FL, Tournel G, Gaulier JM, Marquet P, and Picard N (2013) Involvement of UDP-glucuronosyltransferases UGT1A9 and UGT2B7 in ethanol glucuronidation, and interactions with common drugs of abuse. Drug Metab Dispos 41:568-574

Arnold WR, Weigle AT, and Das A (2018) Cross-talk of cannabinoid and endocannabinoid metabolism is mediated via human cardiac CYP2J2. J Inorg Biochem 184:88-99.

Bland TM, Haining RL, Tracy TS, and Callery PS (2005) CYP2C-catalyzed delta9tetrahydrocannabinol metabolism: kinetics, pharmacogenetics and interaction with phenytoin. Biochem Pharmacol 70:1096-1103.

Bouman HJ, Schömig E, van Werkum JW, Velder J, Hackeng CM, Hirschhäuser C, Waldmann C, Schmalz HG, ten Berg JM, and Taubert D (2011) Paraoxonase-1 is a major determinant of clopidogrel efficacy. Nat Med 17:110-116.

Bridgeman MB and Abazia DT (2017) Medicinal cannabis: history, pharmacology, and implications for the acute care setting. P\&T 42:180-188.

Cerny MA (2016) Prevalence of non-cytochrome P450-mediated metabolism in food and drug administration-approved oral and intravenous drugs: 2006-2015. Drug Metab Dispos 44:1246-1252.

Christophersen AS (1986) Tetrahydrocannabinol stability in whole blood: plastic versus glass containers. J Anal Toxicol 10:129-131.

Desrosiers NA, Himes SK, Scheidweiler KB, Concheiro-Guisan M, Gorelick DA, and Huestis MA (2014) Phase I and II cannabinoid disposition in blood and plasma of occasional and frequent smokers following controlled smoked cannabis. Clin Chem 60:631-643.

Devinsky O, Patel AD, Thiele EA, Wong MH, Appleton R, Harden CL, Greenwood S, Morrison G, and Sommerville K; GWPCARE1 Part A Study Group (2018) Randomized, dose-ranging safety trial of cannabidiol in Dravet syndrome. Neurology 90:e1204-e1211.

Fabritius M, Staub C, Mangin P, and Giroud C (2012) Distribution of free and conjugated cannabinoids in human bile samples. Forensic Sci Int 223:114-118.

Fagerberg L, Hallström BM, Oksvold P, Kampf C, Djureinovic D, Odeberg J, Habuka M, Tahmasebpoor S, Danielsson A, Edlund K, et al. (2014) Analysis of the human tissuespecific expression by genome-wide integration of transcriptomics and antibody-based proteomics. Mol Cell Proteomics 13:397-406.

Fukami T, Takahashi S, Nakagawa N, Maruichi T, Nakajima M, and Yokoi T (2010) In vitro evaluation of inhibitory effects of antidiabetic and antihyperlipidemic drugs on human carboxylesterase activities. Drug Metab Dispos 38:2173-2178.

Garrett ER and Hunt CA (1974) Physiochemical properties, solubility, and protein binding of delta9-tetrahydrocannabinol. J Pharm Sci 63:1056-1064.

Gaston TE, Bebin EM, Cutter GR, Liu Y, and Szaflarski JP; UAB CBD Program (2017) Interactions between cannabidiol and commonly used antiepileptic drugs. Epilepsia 58:1586-1592.

Geffrey AL, Pollack SF, Bruno PL, and Thiele EA (2015) Drug-drug interaction between clobazam and cannabidiol in children with refractory epilepsy. Epilepsia 56:1246-1251.

Gronewold A and Skopp G (2011) A preliminary investigation on the distribution of cannabinoids in man. Forensic Sci Int 210:e7-e11.

Grotenhermen F (2003) Pharmacokinetics and pharmacodynamics of cannabinoids. Clin Pharmacokinet 42:327-360.

He G, Massarella J, and Ward P (1999) Clinical pharmacokinetics of the prodrug oseltamivir and its active metabolite Ro 64-0802. Clin Pharmacokinet 37:471-484

Hoffmann G, Funk C, Fowler S, Otteneder MB, Breidenbach A, Rayner CR, Chu T, and Prinssen EP (2009) Nonclinical pharmacokinetics of oseltamivir and oseltamivir carboxylate in the central nervous system. Antimicrob Agents Chemother 53:4753-4761.

Huestis MA (2007) Human cannabinoid pharmacokinetics. Chem Biodivers 4:1770-1804.

Huestis MA, Henningfield JE, and Cone EJ (1992) Blood cannabinoids. I. Absorption of THC and formation of 11-OH-THC and THCCOOH during and after smoking marijuana. $J$ Anal Toxicol 16:276-282.

Imai T, Taketani M, Shii M, Hosokawa M, and Chiba K (2006) Substrate specificity of carboxylesterase isozymes and their contribution to hydrolase activity in human liver and small intestine. Drug Metab Dispos 34:1734-1741.

Jiang R, Yamaori S, Okamoto Y, Yamamoto I, and Watanabe K (2013) Cannabidiol is a potent inhibitor of the catalytic activity of cytochrome P450 2C19. Drug Metab Pharmacokinet 28:332-338

Johansson E, Ohlsson A, Lindgren JE, Agurell S, Gillespie H, and Hollister LE (1987) Single-dose kinetics of deuterium-labelled cannabinol in man after intravenous administration and smoking. Biomed Environ Mass Spectrom 14:495-499.

Jusko WJ, Gardner MJ, Mangione A, Schentag JJ, Koup JR, and Vance JW (1979) Factors affecting theophylline clearances: age, tobacco, marijuana, cirrhosis, congestive heart failure, obesity, oral contraceptives, benzodiazepines, barbiturates, and ethanol. J Pharm Sci 68:1358-1366.

Jusko WJ, Schentag JJ, Clark JH, Gardner M, and Yurchak AM (1978) Enhanced biotransformation of theophylline in marihuana and tobacco smokers. Clin Pharmacol Ther 24:405-410.
Kosel BW, Aweeka FT, Benowitz NL, Shade SB, Hilton JF, Lizak PS, and Abrams DI (2002) The effects of cannabinoids on the pharmacokinetics of indinavir and nelfinavir. AIDS 16:543-550. Laizure SC, Herring V, Hu Z, Witbrodt K, and Parker RB (2013) The role of human carboxylesterases in drug metabolism: have we overlooked their importance? Pharmacotherapy 33:210-222.

Lee D, Bergamaschi MM, Milman G, Barnes AJ, Queiroz RH, Vandrey R, and Huestis MA (2015) Plasma cannabinoid pharmacokinetics after controlled smoking and ad libitum cannabis smoking in chronic frequent users. $J$ Anal Toxicol 39:580-587.

Lian J, Nelson R, and Lehner R (2018) Carboxylesterases in lipid metabolism: from mouse to human. Protein Cell 9:178-195.

Lindgren JE, Ohlsson A, Agurell S, Hollister L, and Gillespie H (1981) Clinical effects and plasma levels of delta 9-tetrahydrocannabinol (delta 9-THC) in heavy and light users of cannabis. Psychopharmacology (Berl) 74:208-212.

Lucas CJ, Galettis P, and Schneider J (2018) The pharmacokinetics and the pharmacodynamics of cannabinoids. Br J Clin Pharmacol 84:2477-2482.

Mehmedic Z, Chandra S, Slade D, Denham H, Foster S, Patel AS, Ross SA, Khan IA, and ElSohly MA (2010) Potency trends of $\Delta 9$-THC and other cannabinoids in confiscated cannabis preparations from 1993 to 2008. J Forensic Sci 55:1209-1217.

Newmeyer MN, Swortwood MJ, Barnes AJ, Abulseoud OA, Scheidweiler KB, and Huestis MA (2016) Free and glucuronide whole blood cannabinoids' pharmacokinetics after controlled smoked, vaporized, and oral cannabis administration in frequent and occasional cannabis users: identification of recent cannabis intake. Clin Chem 62:1579-1592.

Ohlsson A, Lindgren JE, Andersson S, Agurell S, Gillespie H, and Hollister LE (1986) Single-dose kinetics of deuterium-labelled cannabidiol in man after smoking and intravenous administration. Biomed Environ Mass Spectrom 13:77-83.

Perez-Reyes M, Di Guiseppi S, Davis KH, Schindler VH, and Cook CE (1982) Comparison of effects of marihuana cigarettes to three different potencies. Clin Pharmacol Ther 31:617-624.

Rhoades JA, Peterson YK, Zhu HJ, Appel DI, Peloquin CA, and Markowitz JS (2012) Prediction and in vitro evaluation of selected protease inhibitor antiviral drugs as inhibitors of carboxylesterase 1: a potential source of drug-drug interactions. Pharm Res 29:972-982.

Ross MK and Crow JA (2007) Human carboxylesterases and their role in xenobiotic and endobiotic metabolism. J Biochem Mol Toxicol 21:187-196.

Roth MD, Marques-Magallanes JA, Yuan M, Sun W, Tashkin DP, and Hankinson O (2001) Induction and regulation of the carcinogen-metabolizing enzyme CYP1A1 by marijuana smoke and delta (9)-tetrahydrocannabinol. Am J Respir Cell Mol Biol 24:339-344.

Satoh T and Hosokawa M (2006) Structure, function and regulation of carboxylesterases. Chem Biol Interact 162:195-211

Satoh T, Taylor P, Bosron WF, Sanghani SP, Hosokawa M, and La Du BN (2002) Current progress on esterases: from molecular structure to function. Drug Metab Dispos 30:488-493.

Schwope DM, Karschner EL, Gorelick DA, and Huestis MA (2011) Identification of recent cannabis use: whole-blood and plasma free and glucuronidated cannabinoid pharmacokinetics following controlled smoked cannabis administration. Clin Chem 57:1406-1414.

Segel IH (1975) Enzyme Kinetics: Behavior and Analysis of Rapid Equilibrium and Steady State Enzyme Systems, John Wiley \& Sons, Inc, Hoboken, NJ.

Takai S, Matsuda A, Usami Y, Adachi T, Sugiyama T, Katagiri Y, Tatematsu M, and Hirano K (1997) Hydrolytic profile for ester- or amide-linkage by carboxylesterases pI 5.3 and 4.5 from human liver. Biol Pharm Bull 20:869-873.

Taylor L, Gidal B, Blakey G, Tayo B, and Morrison G (2018) A phase I, randomized, double-blind, placebo-controlled, single ascending dose, multiple dose, and food effect trial of the safety, tolerability and pharmacokinetics of highly purified cannabidiol in healthy subjects. CNS Drugs 32:1053-1067.

Thomsen R, Rasmussen HB, and Linnet K; INDICES Consortium (2014) In vitro drug metabolism by human carboxylesterase 1: focus on angiotensin-converting enzyme inhibitors. Drug Metab Dispos 42:126-133.

Wang DD, Zou LW, Jin Q, Hou J, Ge GB, and Yang L (2017) Recent progress in the discovery of natural inhibitors against human carboxylesterases. Fitoterapia 117:84-95.

Williams JA, Hyland R, Jones BC, Smith DA, Hurst S, Goosen TC, Peterkin V, Koup JR, and Bal SE (2004) Drug-drug interactions for UDP-glucuronosyltransferase substrates: a pharmacokinetic explanation for typically observed low exposure (AUCi/AUC) ratios. Drug Metab Dispos 32:1201-1208.

Yamaori S, Ebisawa J, Okushima Y, Yamamoto I, and Watanabe K (2011a) Potent inhibition of human cytochrome P450 3A isoforms by cannabidiol: role of phenolic hydroxyl groups in the resorcinol moiety. Life Sci 88:730-736.

Yamaori S, Koeda K, Kushihara M, Hada Y, Yamamoto I, and Watanabe K (2012) Comparison in the in vitro inhibitory effects of major phytocannabinoids and polycyclic aromatic hydrocarbons contained in marijuana smoke on cytochrome P450 2C9 activity. Drug Metab Pharmacokinet 27:294-300.

Yamaori S, Kushihara M, Yamamoto I, and Watanabe K (2010) Characterization of major phytocannabinoids, cannabidiol and cannabinol, as isoform-selective and potent inhibitors of human CYP1 enzymes. Biochem Pharmacol 79:1691-1698.

Yamaori S, Maeda C, Yamamoto I, and Watanabe K (2011b) Differential inhibition of human cytochrome P450 2A6 and 2B6 by major phytocannabinoids. Forensic Toxicol 29:117-124.

Yamaori S, Okamoto Y, Yamamoto I, and Watanabe K (2011c) Cannabidiol, a major phytocannabinoid, as a potent atypical inhibitor for CYP2D6. Drug Metab Dispos 39:2049-2056.

Zhu HJ, Appel DI, Peterson YK, Wang Z, and Markowitz JS (2010) Identification of selected therapeutic agents as inhibitors of carboxylesterase 1: potential sources of metabolic drug interactions. Toxicology 270:59-65.

Zhu HJ, Patrick KS, Yuan HJ, Wang JS, Donovan JL, DeVane CL, Malcolm R, Johnson JA, Youngblood GL, Sweet DH, et al. (2008) Two CES1 gene mutations lead to dysfunctional carboxylesterase 1 activity in man: clinical significance and molecular basis. Am J Hum Genet 82:1241-1248

Address correspondence to: John S. Markowitz, Department of Pharmacotherapy and Translational Research, University of Florida College of Pharmacy, 1600 SW Archer Road, RM PG-23, Gainesville, FL 32610-0486. E-mail: jmarkowitz@cop.ufl.edu 